\title{
Chromosome copy number changes carry prognostic information independent of KIT/PDGFRA point mutations in gastrointestinal stromal tumors
}

\author{
Mara Silva1, Isabel Veiga1, Franclim R Ribeiro', Joana Vieira1, Carla Pinto', Manuela Pinheiro1, Bárbara Mesquita1, \\ Catarina Santos ${ }^{1}$, Marta Soares², José Dinis², Lúcio Santos³, Paula Lopes ${ }^{4}$, Mariana Afonso ${ }^{4}$, Carlos Lopes ${ }^{4}$ and \\ Manuel R Teixeira*1,5
}

\begin{abstract}
Background: Oncogenic point mutations in KIT or PDGFRA are recognized as the primary events responsible for the pathogenesis of most gastrointestinal stromal tumors (GIST), but additional genomic alterations are frequent and presumably required for tumor progression. The relative contribution of such alterations for the biology and clinical behavior of GIST, however, remains elusive.
\end{abstract}

Methods: In the present study, somatic mutations in KIT and PDGFRA were evaluated by direct sequencing analysis in a consecutive series of 80 GIST patients. For a subset of 29 tumors, comparative genomic hybridization was additionally used to screen for chromosome copy number aberrations. Genotype and genomic findings were cross-tabulated and compared with available clinical and follow-up data.

Results: We report an overall mutation frequency of $87.5 \%$, with $76.25 \%$ of the tumors showing alterations in KIT and $11.25 \%$ in PDGFRA. Secondary KIT mutations were additionally found in two of four samples obtained after imatinib treatment. Chromosomal imbalances were detected in 25 out of 29 tumors (86\%), namely losses at $14 q$ ( $88 \%$ of abnormal cases), 22q (44\%), $1 p(44 \%)$, and 15q (36\%), and gains at $1 q(16 \%)$ and $12 q(20 \%)$. In addition to clinicopathological high-risk groups, patients with KIT mutations, genomic complexity, genomic gains and deletions at either $1 p$ or $22 q$ showed a significantly shorter disease-free survival. Furthermore, genomic complexity was the best predictor of disease progression in multivariate analysis.

Conclusions: In addition to KIT/PDGFRA mutational status, our findings indicate that secondary chromosomal changes contribute significantly to tumor development and progression of GIST and that genomic complexity carries independent prognostic value that complements clinico-pathological and genotype information.

\section{Background}

Gastrointestinal stromal tumors (GIST) represent the most common mesenchymal tumors of the gastrointestinal tract [1]. A diagnosis of GIST involves a multidisciplinary approach that combines clinical, pathological, and genetic features. Mutually exclusive activating mutations in KIT or PDGFRA occur in 85 to $90 \%$ of the cases

* Correspondence: manuel.teixeira@ipoporto.min-saude.pt

${ }^{1}$ Department of Genetics, Portuguese Oncology Institute - Porto, Rua Dr. António Bernardino Almeida, 4200-072 Porto, Portugal

Full list of author information is available at the end of the article and are considered primary events in GIST pathogenesis [1-3]. These genes encode type III transmembrane receptor proteins which, upon connection to their respective ligands, activate downstream signaling pathways involved in cell proliferation and survival [4-6].

Whereas oncogenic KIT or PDGFRA mutations seem vital to promote the neoplastic transformation, additional somatic alterations are presumably necessary for the biological and clinical progression of these tumors and may explain the different responses to targeted therapy seen in these patients. Genome-screening methodologies, such 
as conventional cytogenetics and comparative genomic hybridization (CGH), have been applied in order to identify these changes. Some chromosomal alterations, such as losses at 1p, 14q, and 22q, are particularly frequent, suggesting the existence of tumor suppressor genes in these regions that could be important in tumor progression [7-10]. Although this cytogenetic fingerprint of GIST has been defined, the target genes involved in these regions remain undiscovered [11]. Furthermore, the relationship between the pattern of KIT and PDGFRA oncogenic mutations and that of cytogenetic changes has not been systematically studied, precluding a full understanding of the genetic pathways involved in GIST development.

In this work, we assessed the genetic background of a consecutive series of 80 patients diagnosed with GIST. KIT or PDGFRA mutations were evaluated in all samples using direct sequencing analysis. For a subset of 29 patients with fresh-frozen tisue, CGH was used to screen for chromosomal copy number aberrations. Cytogenetic and molecular genetic findings were integrated and correlated with clinico-pathological parameters, including imatinib/sunitinib therapy response.

\section{Methods}

\section{Clinical samples}

A series of 80 patients diagnosed with GIST and submitted to surgery with curative intent were included in this study. The majority of patients was diagnosed and treated at the Portuguese Oncology Institute - Porto, with the exception of six cases that were provided by other institutions. Patients had received no treatment prior to surgery. Fresh-frozen tumor samples from 29 patients were available for mutational and CGH analyses, whereas for the remaining cases mutational analyses were performed in formalin-fixed, paraffin-embedded tissue sections. In all cases, hematoxylin and eosin stained sections from representative tissue blocks were reviewed by expert pathologists to confirm a diagnosis of GIST and to evaluate relevant histopathological parameters. Immunohistochemistry for CD117 followed the standard avidin-biotin-peroxidase complex method with a commercial polyclonal antibody at a 1/600 dilution (A4502, Dako, Glostrup, Denmark). Other clinical and demographic variables, such as age at diagnosis, gender, tumor size, and tumor location (divided into stomach, small intestine, rectum and colon, and outside the GI tract), were obtained Additional file 1. Patients with tumors that eventually recurred or that developed metastatic lesions $(\mathrm{n}=36)$ were treated with imatinib in accordance with the guidelines followed at the IPO-Porto. Second-line therapy for patients that progressed or were intolerant to imatinib $(\mathrm{n}=2)$ was sunitinib. This study was approved by the institutional review board and was performed in accordance with national regulations (law 12/2005).

\section{KIT and PDGFRA mutation screening}

DNA isolation from formalin-fixed, paraffin-embedded tumor samples and from physically disaggregated freshfrozen tissue fragments was performed using an adaptation of the technique described by Lungu and colleagues [12] or a salting-out-chloroform mixed methodology [13], respectively. Using the DNA extracted from each sample, KIT (exons 9, 11,13, and 17) and PDGFRA (exons 12,14 , and 18) target sequences were amplified by polymerase chain reaction (PCR) on a standard termocycler. Primers and conditions were as described in the literature $[14,15]$. Direct sequencing was performed on an ABI PRISM 310 automatic sequencer using the Big Dye Terminator Chemistry (Applied Biosystems, Foster City, CA, USA), according to the manufacturer's recommendations. All results were confirmed with a second independent analysis.

\section{Comparative genomic hybridization}

Fresh-frozen tumor samples from 29 patients were analyzed by CGH following the procedure of Kallioniemi et al. [16], with modifications previously described [17]. Samples were analyzed with a Cohu 4900 CCD camera using an automated filter wheel coupled to a Zeiss Axioplan fluorescence microscope (Zeiss, Oberkochen, Germany) and a Citovysion system version 3.9 (Applied Imaging, Santa Clara, CA, USA). For each sample, data from 10 cells were combined to generate average ratio profiles with $99 \%$ confidence intervals and aberrations were scored whenever the sample profile and the standard reference profile at $99 \%$ did not overlap [18]. Description of the CGH copy number changes followed the guidelines suggested by the International System for Human Cytogenetic Nomenclature (ISCN) 2005 [19].

\section{Statistical analysis}

Relevant clinico-pathological (gender, age, tumor size, tumor location and patient risk groups) and genetic (mutation status and chromosomal imbalances) variables were cross-tabulated and analyzed using the chi-square or Fisher's exact test. The number of chromosomal aberrations was compared within groups of samples with different mutation genotypes using the non-parametric Mann-Whitney U test. Kaplan-Meyer survival curves using log-rank test were computed for relevant clinical and genetic events. For statistical purposes, patients with recurrent or metastatic lesions were included in the highrisk group. A $P$-value lower than 0.05 was considered statistically significant. All analysis was performed using the Statistical Package for Social Sciences (SPSS) software, version 15 (SPSS Inc., Chicago, IL, USA). 


\section{Results}

Clinicopathologic characteristics of the patients

A total of 80 patients diagnosed with GIST were enrolled in this study. Tumor location was obtainable in 79 cases, of which 67 corresponded to primary lesions Additional file 1 . Twelve recurrent or metastatic lesions were analyzed due to lack of the primary sample. For four of the patients, a second sample collected after disease progression could additionally be assessed, increasing the number of lesions submitted to sequencing analysis to 84 . Tumor size was recorded in 70 cases and varied from 1.2 to $45 \mathrm{~cm}$ (average $8.8 \mathrm{~cm}$ ). From the available morphology data, the series included 52 spindle cell tumors, 6 epithelioid lesions, and 13 mixed tumors. Based on the National Comprehensive Cancer Network taskforce guidelines for GIST risk assessment [20], most tumors in this series could be classified as either low/very low risk $(\mathrm{n}=27)$, moderate risk $(\mathrm{n}=8)$, or high risk $(\mathrm{n}=27)$. Expression of the KIT protein (CD117) was assessed in 74 cases. A total of 70 lesions (94.6\%) showed a positive staining pattern, whereas two cases were negative and two cases presented inconclusive findings.

\section{KIT and PDGFRA mutations}

Samples from all 80 patients were screened for mutations within exons $9,11,13$, and 17 of the oncogene KIT. Mutations were detected in 61 tumors $(76.25 \%$, Figure 1), namely in exon $11(n=52)$, exon $9(n=7)$ and exon $17(n$ $=2$ ). The two patients with KIT exon 17 mutation were subsequently found to be relatives and the mutation shown to be present in the germline [21]. No primary mutations were found in exon 13. All KIT negative cases

( $\mathrm{n}=19$ ) were then analyzed for mutations in exons 12,14 , and 18 of PDGFRA. A total of nine samples (11.25\%) showed mutations in this gene, namely in exon $18(n=6)$, exon $12(\mathrm{n}=2)$, and exon $14(\mathrm{n}=1)$. CD117 staining was seen in six out of eight PDGFRA positive cases. The overall mutation frequency for both genes in this series was $87.5 \%$ (70 out of 80 tumors). Of note, two tumors with KIT exon 11 primary mutations and with an initial response to imatinib, acquired resistance and developed peritoneal or hepatic metastases that presented the same secondary mutation (KIT exon 13, p.Val654Glu). A complete description of the mutations and relevant clinical parameters for each patient are detailed in Additional file 1.

\section{Chromosome copy number changes}

Out of the 29 GIST submitted to whole-genome screening, 25 (86\%) displayed copy number changes (Figure 2, Additional file 2). Most abnormal samples displayed noncomplex profiles, with a median of three aberrations per tumor (ranging from one to 28 changes), and losses were 1.5 times more frequent than gains. It is noteworthy that complete or partial loss of $14 \mathrm{q}$ was seen in 22 samples (88\%), being the sole copy number change in four patients. Other frequent changes included losses at $22 \mathrm{q}$ (44\%), $1 \mathrm{p}(44 \%)$, and $15 \mathrm{q}(36 \%)$ and gains at $1 \mathrm{q}(16 \%)$ and $12 \mathrm{q}(20 \%)$. All 25 cytogenetically abnormal GIST presented at least one of the losses 1p, 14q, or 22q.

\section{Integrative analysis of molecular and cytogenetic alterations}

Based on previous literature findings, samples submitted to $\mathrm{CGH}$ analysis were divided according to mutation gen-

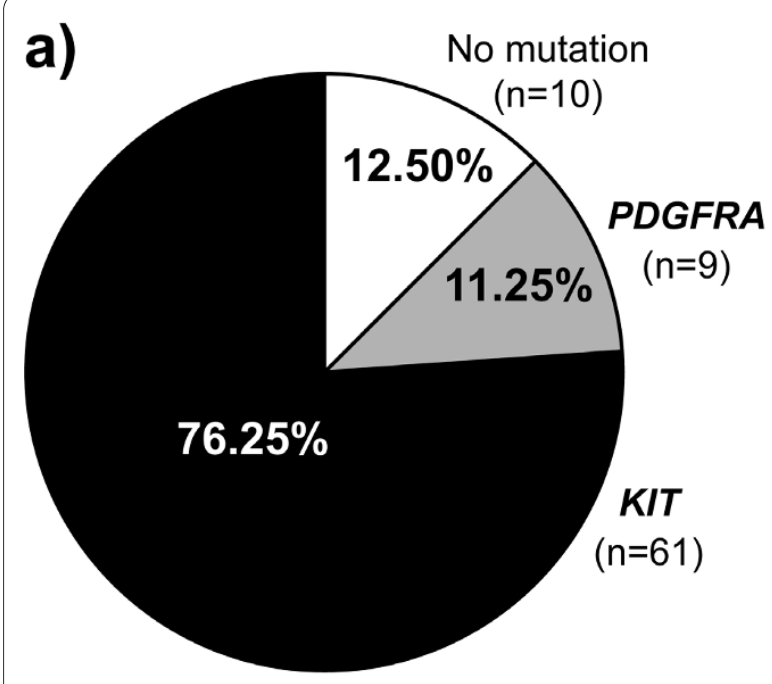

b)
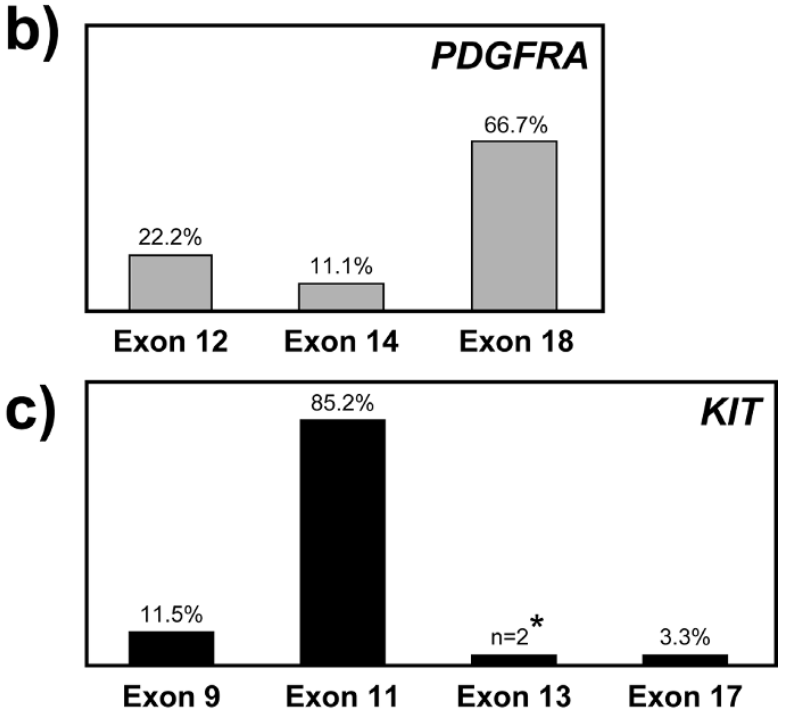

Figure 1 Mutation profile of KIT and PDGFRA in $\mathbf{8 0}$ GIST. a) mutation frequency per gene; b) mutation frequency per PDGFRA exon; $\mathbf{c}$ ) mutation frequency per KIT exon. Two patients with primary mutations in KIT exon 11 acquired the same secondary mutation in KIT exon 13 after imatinib treatment. The two patients with KIT exon 17 mutation belong to a family with hereditary GIST, with the mutation being found also in the germline. 


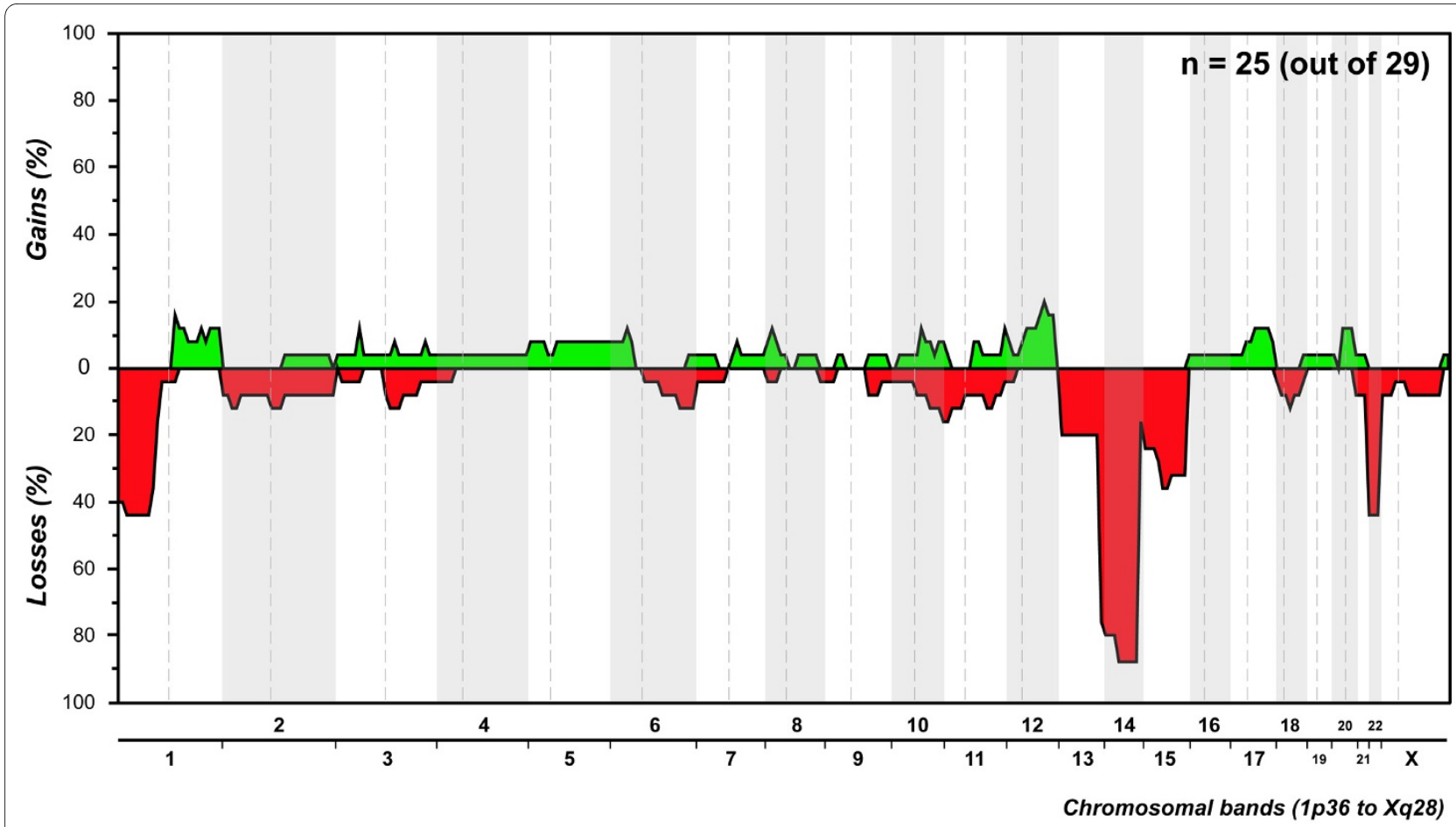

Figure 2 Copy number profile of patients diagnosed with GIST. Gains and losses of genetic material are depicted along all chromosomes (X axis).

otypes to test for possible correlations. Genomic results were compared between samples with $K I T$ exon 9 mutations $(\mathrm{n}=3)$, KIT exon 11 deletions/delins $(\mathrm{n}=8)$ or samples with no detectable mutations $(\mathrm{n}=1)$, totaling 12 cases associated in the literature with bad prognosis, versus samples with KIT or PDGFRA mutations not previously associated with a worse prognosis $(\mathrm{n}=13)$. Strikingly, the former group showed significantly more copy number changes than the latter (median of 6.5 versus 2 aberrations per tumor, $P=0.026$, Mann-Whitney $\mathrm{U}$ test). The three cases with KIT exon 9 mutations showed the most complex CGH profiles (median of nine aberrations per tumor), followed by those with exon 11 deletions/delins (median of four aberrations per tumor). It is noteworthy that three of the four cases without detectable CGH alterations showed no mutations in either KIT or PDGFRA. No significant associations were observed between specific copy number changes and different mutation subgroups. Tumors with PDGFRA mutations showed the same overall pattern of alterations seen in those with KIT mutations, even if genomic complexity was much lower in the former (median of 2 vs. 5.5 alterations per tumor, respectively, $P=0.050$, Mann-Whitney U test).

\section{Therapeutic correlations and survival data}

Follow-up data was available in 74 cases (median of 30 months, ranging from 8 to 123 months). During this period, 26 patients (35\%) showed disease progression and were subsequently treated with imatinib. According with the most recent clinical records, six of these patients died from their cancer. Most of the samples from the progression group showed KIT mutations, namely in exon 11 (n $=21$ ) and exon $9(\mathrm{n}=3)$, with only two patients showing no mutations in either gene. The 48 patients that received no adjuvant therapy are currently alive without evidence of disease, with the exception of three non disease-related deaths. Within this group, 41 tumors harbored mutations, namely in KIT exon $11(\mathrm{n}=28), K I T$ exon $9(\mathrm{n}=4)$, PDGFRA exon $12(\mathrm{n}=2)$, PDGFRA exon $14(\mathrm{n}=1)$, and PDGFRA exon $18(\mathrm{n}=6)$.

Disease-specific survival curves were uninformative due to the reduced number of death-from-disease events, and five-year disease-free survival curves were thus computed (Figures 3 and 4, Additional file 3). Stratification according to tumor location showed that lesions in the stomach progressed much less frequently than those in other locations $(P<0.001$, Figure 3a). Regarding risk groups, most progression events were seen in lesions categorized as high risk $(P=0.001$, Figure $3 \mathrm{~b})$. When patients were categorized based on genetic variables, a more aggressive outcome was seen in patients with $K I T$ mutations compared to those with PDGFRA mutations $(P=0.039$, Figure 4a). Based on previous literature reports, patients were additionally categorized according to specific mutations associated with worse prognosis. Patients with KIT exon 9 or KIT exon 11 deletions/delins 

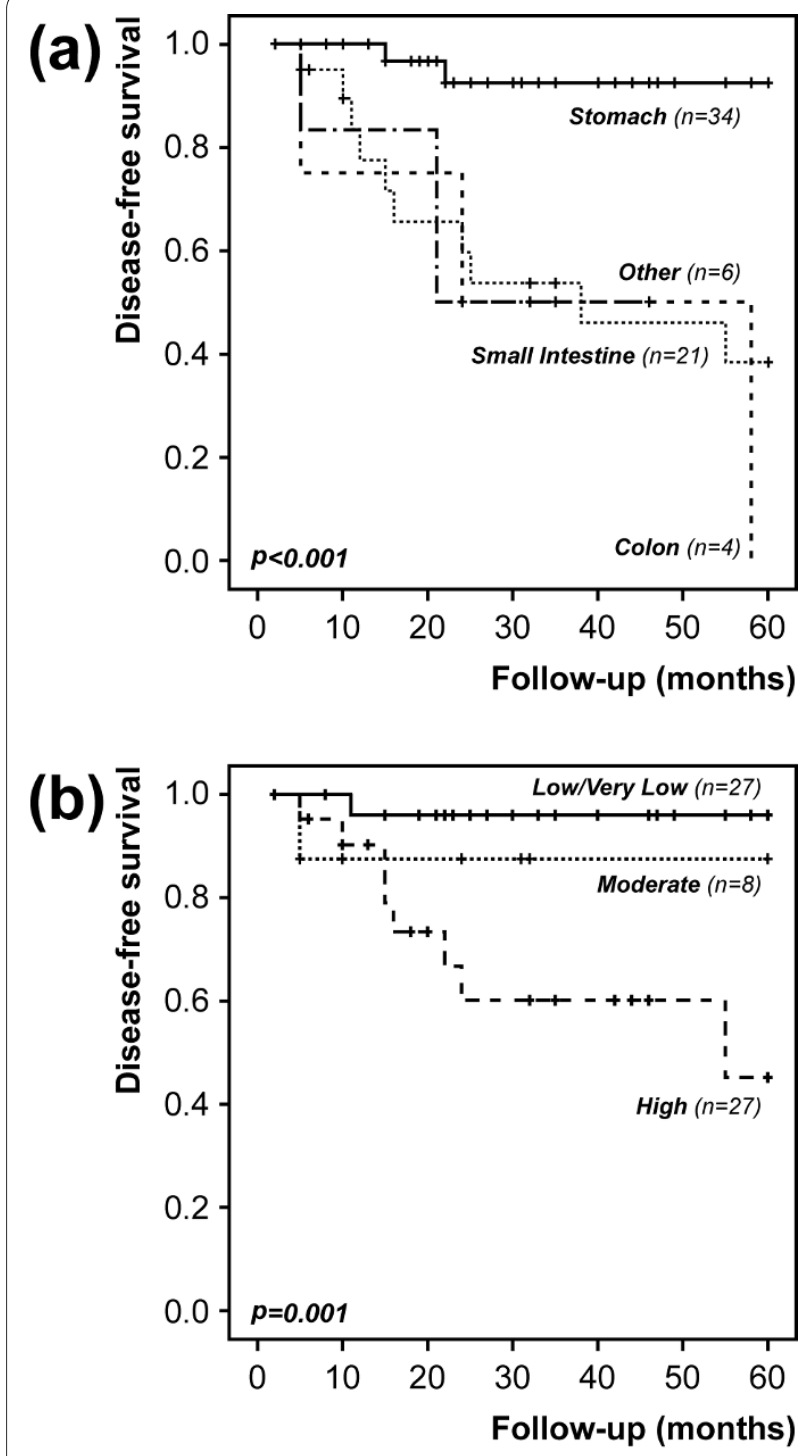

Figure 3 Five-year disease-free survival curves based on selected clinical variables. a) Tumor location; b) Risk groups.

( $\mathrm{n}=25$ cases) showed a tendentiously worse progressionfree survival $(P=0.091)$ than those showing mutations in PDGFRA or other mutations in KIT ( $\mathrm{n}=33$ cases). Within the subgroup of patients with KIT exon 11 mutations, the number of progression events in tumors with deletions/delins was significantly higher than those with other mutations $(P=0.003$, Fisher's test). In multivariate analysis including risk groups and genotype (KIT vs PDGFRA mutations), a high risk at diagnosis was the strongest predictor of relapse (HR $=9.8, P=0.003,95 \% \mathrm{CI}=2.2$ to 43.1) (Additional file 4: Supplementary Table 4a)

In the subgroup of patients with CGH data and complete follow-up information $(n=27)$, genomic complexity was very strongly associated with a worse outcome $(P=$ 0.002 , Figure $4 \mathrm{~b})$. The presence of genomic gains $(P=$
$0.006)$ or deletions at $1 \mathrm{p}$ or $22 \mathrm{q}(P=0.030)$ were also significantly associated with a shorter progression-free period (Figure 4c and 4d, Additional file 3). Multivariate survival analysis in this subset (using risk groups, genotype status, genomic gains and losses at $1 \mathrm{p}$ or $22 \mathrm{q}$ ) showed that the best predictor of progression was genomic complexity (HR $=13.7, P=0.014,95 \% \mathrm{CI}=1.7$ to 111.1, Additional file 4: Supplementary Table 4b).

\section{Discussion}

Recent years have seen important breakthroughs that resulted in better diagnostic, prognostic and therapeutic tools for patients with GIST. Identification of a distinctive molecular signature involving KIT or PDGFRA mutations allowed targeted therapies in patients with metastatic disease, who did not have effective therapeutic options until then. However, additional genomic changes are known to occur in GIST that might influence therapy response and tumor aggressiveness. In the current work, we characterized the mutation profile of KIT and PDFGRA in a consecutive series of GIST diagnosed and followed at our institution. Gross genomic aberrations were additionally assessed in a subset of these patients, in order to determine the relative contribution of primary and secondary genetic events in GIST as prognostic or/and predictive factors.

Tumor size and mitotic index have been considered the most important prognostic indicators in GIST [22,23]. However, it has been shown that even small GIST can behave aggressively and develop metastases. Indeed, one patient with an intestinal lesion with less than $2 \mathrm{~cm}$ and low mitotic rate (patient 20, categorized in the low-risk group) developed metastases and died from the disease 11 months after diagnosis. More recently, anatomic location was also considered of relevance and included in the determination of the risk of recurrence and progression $[21,24]$. Our findings strongly support this prediction model, as a significant proportion of small intestine or colon GIST developed metastasis, whereas most tumors located in the stomach showed no progression events.

KIT and PDGFRA activating mutations are mutually exclusive events in GIST that promote the constitutive activation of the receptors and the downstream signaling pathways, resulting in aberrant cell proliferation and apoptosis [2]. The overall frequency of KIT and PDGFRA mutations in GIST varies in different studies, but is usually higher than $80 \%$ [25]. In our 80 samples, we obtained a mutation frequency of $87.2 \%$, with $75.7 \%$ of the cases harboring KIT mutations and $11.5 \%$ showing PDGFRA mutations, which is significantly higher than the 63\% recently found in a second Portuguese series of GIST [26] and that of another Iberian Peninsula series [27]. It has been suggested that the type and molecular location of different mutational events in GIST carry distinct biolog- 

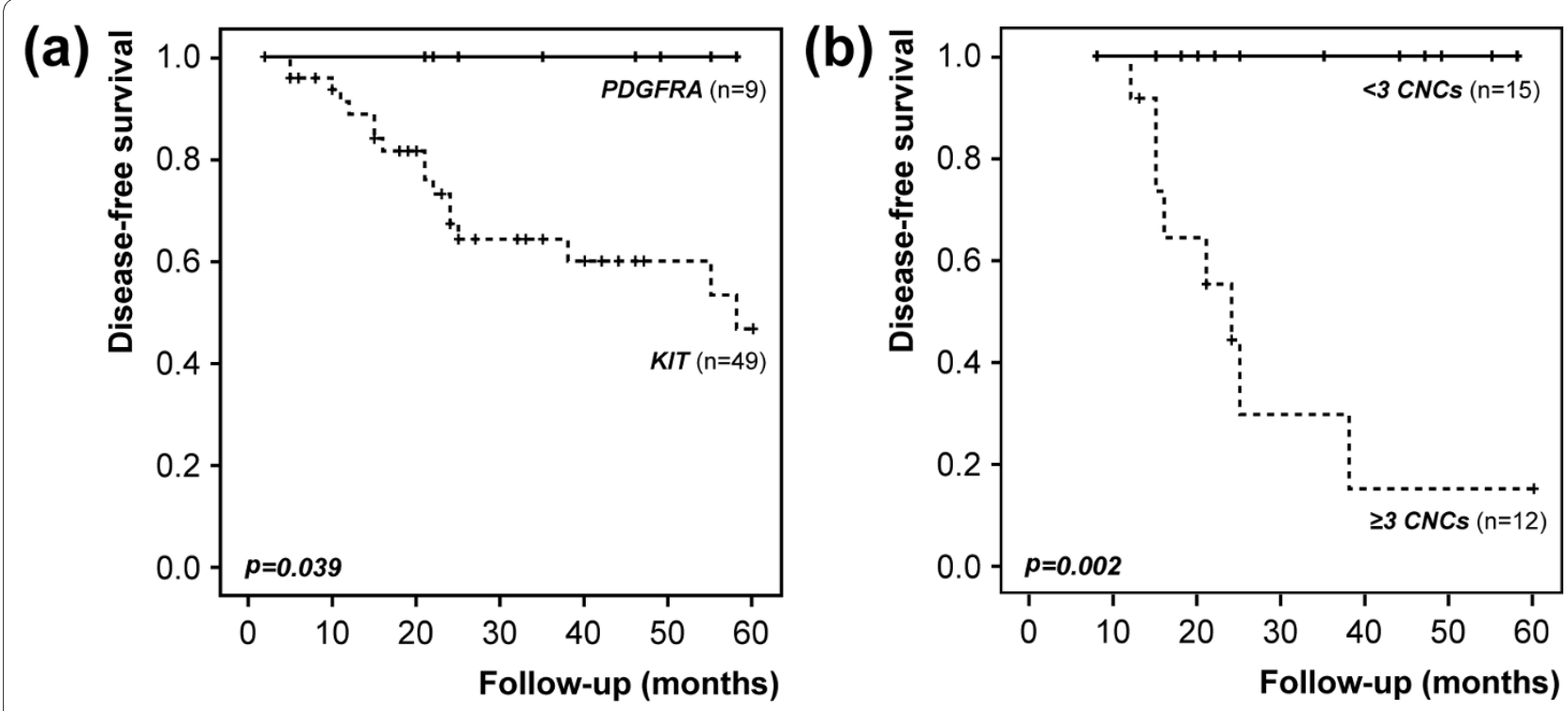
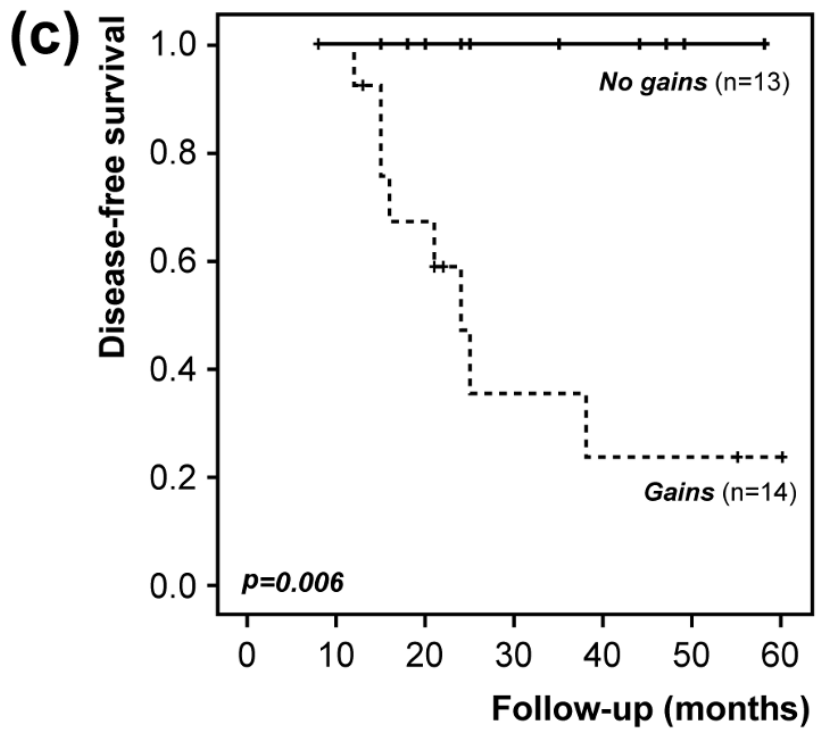

(d)

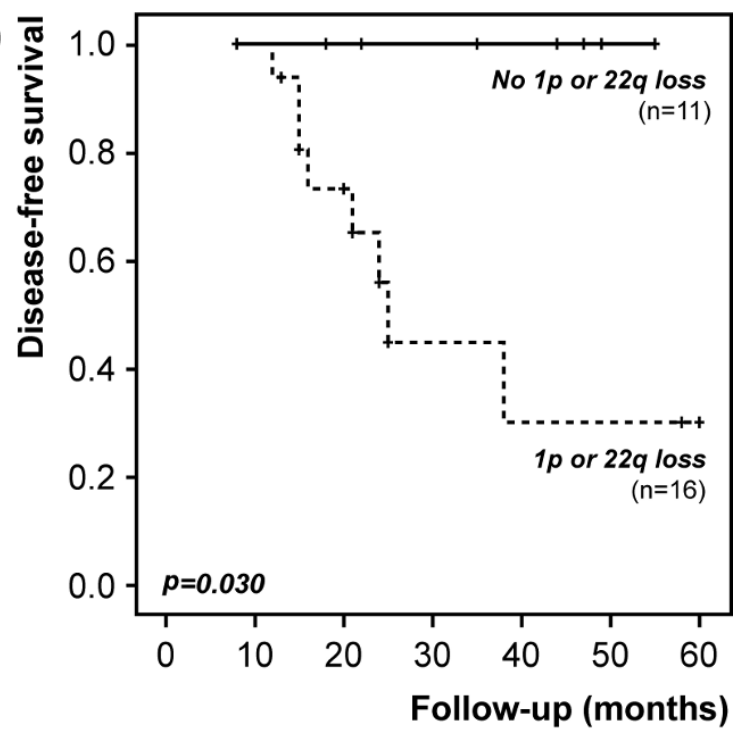

Figure 4 Five-year disease-free survival based on selected genetic variables. a) Mutated gene; b) genomic complexity; c) genomic gains; d) $1 p$ or 22q deletions.

ical and clinical implications [1,28]. Mutations in the KIT extracellular regulatory domain, coded by exon 9 , seem to mimic the conformational changes that follow stem-cell factor (SCF) ligation. The most common mutation found within this location (p.Ala502_Tyr503dup) corresponds to an insertion of six nucleotides [29], and indeed all our tumors with exon 9 mutations displayed this hot-spot alteration. The major mutational hotspot in KIT is in exon 11, which encodes the juxtamembrane intracellular domain responsible for modulating KIT enzymatic activity [24]. KIT exon 11 deletions have been linked to an aggressive behavior comparing missense and insertion mutations $[27,28,30,31]$. In our series, 26 out of 52 muta- tions in this domain corresponded to deletions/delins. Interestingly, 15 of these 26 patients showed disease progression, whereas only four patients in the group with insertions, duplications or missense mutations showed disease progression $(P=0.003)$. It is noteworthy that in two patients with primary $K I T$ exon 11 mutations treated with imatinib and in whom additional metastases developed, the same secondary mutation in KIT exon 13 (p.Val654Glu), known to confer imatinib resistance [32], was detected. Finally, the two patients in which we identified the same KIT exon 17 mutation were recently found to be relatives. In fact, we were able to show that the p.Asp820Tyr was present also in the germline, represent- 
ing the third example in the literature of hereditary GIST caused by this same mutation [21].

GIST harboring PDGFRA mutations share many clinical features with KIT mutated tumors, but are mainly gastric and present weak or negative CD117 staining [33]. Existent evidence suggests that PDGFRA mutated tumors might be less aggressive [34]. Of the nine cases with $P D G$ FRA mutations in our series, three showed weak or only focal CD117 staining, seven were located in the stomach, and all patients are currently alive with no evidence of disease. Interestingly, the hotspot p.Asp842Val mutation, which has been associated with primary resistance to imatinib [3], was detected in four of these cases. However, these four patients were classified in the low risk group and showed no signs of progression thus far. As such, they have not been submitted to imatinib treatment.

In addition to the primary mutation events activating KIT or PDGFRA, cytogenetic studies have shown additional changes associated with GIST progression $[7,35,36]$. However, few studies so far have performed genotype and genome analysis in the same samples, preventing a reliable assessment of correlations between primary and secondary genetic events, or their combined prognostic/predictive value [37-39]. In our work, $86 \%$ of the GIST submitted to CGH analysis displayed copy number changes. Complete or partial deletions of chromosome 14 were seen in $88 \%$ of the abnormal cases, and in four patients this was the sole chromosomal change detected. Additional recurrent cytogenetic aberrations included losses at $22 \mathrm{q}, 1 \mathrm{p}$, and $15 \mathrm{q}$, as well as gains at $1 \mathrm{q}$ and 12q. Genomic complexity (three or more aberrations per tumor), the presence of gains, deletions at $1 \mathrm{p}$, and deletions at $22 q$ were associated with a shorter diseasefree survival in this subset of patients, with multivariate analysis evidencing genomic complexity as the best predictor of disease relapse. Strikingly, tumors harboring KIT mutations associated with a bad prognosis showed significantly more chromosome copy number changes than those without such mutations. On the opposite side, tumors with PDGFRA mutations showed the same overall pattern of alterations seen in those with KIT mutations, but the complexity was much lower and no progression events were observed.

\section{Conclusions}

Taken together, our findings suggest that secondary chromosome changes have independent prognostic value in GIST. Furthermore, chromosome level information might also be useful for differential diagnosis, as the pattern of genomic losses of $1 \mathrm{p}, 14 \mathrm{q}$, and/or $22 \mathrm{q}$ is rather characteristic. Additional integrative molecular and cytogenetic studies are necessary to assess the combined diagnostic, prognostic, and predictive value of the several recurrent genetic features of GIST.

\section{Additional material}

\begin{abstract}
Additional file 1 Clinicopathologic characteristics and genotypic results of $\mathbf{8 0}$ patients diagnosed with GIST. Table summarizing clinical and genotypic variables of the 80 patients enrolled in this study.

Additional file $\mathbf{2}$ Chromosomal imbalances and molecular alterations detected in 29 GIST submitted to CGH analysis. Table integrating CGH and genotypic data for 29 patients for which both information was available.

Additional file 3 Disease-free survival results for selected variables. Univariate disease-free survival results for selected genetic and clinical variables, using the Kaplan-Meyer test with Log-rank statistics at 60 months follow-up.

Additional file $\mathbf{4}$ Multivariate disease-free survival results. Results of the cox-regression models (forward conditional setting) using the variables that showed significant prognostic value in univariate testing.
\end{abstract}

\section{Abbreviations}

CCD: charge-coupled device; CGH: comparative genomic hybridization; Gl: gastrointestinal; GIST: gastrointestinal stromal tumors; HR: hazard ratio; ISCN: international system for cytogenetic nomenclature; PCR: polymerase chain reaction; SCF: stem cell factor; SPSS: statistical package for social sciences.

\section{Competing interests}

The authors declare that they have no competing interests.

\section{Authors' contributions}

MSi, IV, CP, MP, BM and CS carried out DNA extractions and genotype analysis and interpretations. MSi and JV performed molecular cytogenetic analysis. FRR carried out statistical analysis and drafted the manuscript together with MSi. MSo, JD and LS provided patient clinical data. PL, MA and CL carried out pathological assessment of the tumors. MRT designed and coordinated the study, assisted with scoring the molecular cytogenetic changes, and contributed to manuscript writing. All authors read and approved the final manuscript.

\section{Acknowledgements}

This work was in part supported by Liga Portuguesa Contra o Cancro - Núcleo Regional do Norte. BM and FRR are research fellows of Fundação para a Ciência e a Tecnologia.

\section{Author Details}

1Department of Genetics, Portuguese Oncology Institute - Porto, Rua Dr. António Bernardino Almeida, 4200-072 Porto, Portugal, 2Department of Oncology, Portuguese Oncology Institute - Porto, Rua Dr. António Bernardino Almeida, 4200-072 Porto, Portugal, ${ }^{3}$ Department of Surgery, Portuguese Oncology Institute - Porto, Rua Dr. António Bernardino Almeida, 4200-072 Porto, Portugal, ${ }^{4}$ Department of Pathology, Portuguese Oncology Institute Porto, Rua Dr. António Bernardino Almeida, 4200-072 Porto, Portugal and 5Institute of Biomedical Sciences Abel Salazar (ICBAS), University of Porto, Largo Prof. Abel Salazar, 4099-003 Porto, Portugal

Received: 21 April 2010 Accepted: 14 May 2010

Published: 14 May 2010

\section{References}

1. Corless CL, Heinrich MC: Molecular pathobiology of gastrointestinal stromal sarcomas. Annu Rev Pathol 2008, 3:557-586.

2. Hirota S, Isozaki K, Moriyama Y, Hashimoto K, Nishida T, Ishiguro S, Kawano K, Hanada M, Kurata A, Takeda M, Muhammad TG, Matsuzawa Y, Kanakura $Y$, Shinomura $Y$, Kitamura $Y$ : Gain-of-function mutations of c-kit in human gastrointestinal stromal tumors. Science 1998, 279:577-580.

3. Heinrich MC, Corless CL, Duensing A, McGreevey L, Chen CJ, Joseph N, Singer S, Griffith DJ, Haley A, Town A, Demetri GD, Fletcher CD, Fletcher JA: PDGFRA activating mutations in gastrointestinal stromal tumors. Science 2003, 299:708-710.

4. Taylor ML, Metcalfe DD: Kit signal transduction. Hematol Oncol Clin North Am 2000, 14:517-535. 
5. Savage DG, Antman KH: Imatinib mesylate - a new oral targeted therapy. NEng/ J Med 2002, 346:683-693.

6. Xiang Z, Kreisel F, Cain J, Colson A, Tomasson MH: Neoplasia driven by mutant c-KIT is mediated by intracellular, not plasma membrane, receptor signaling. Mol Cell Biol 2007, 27:267-282.

7. El Rifai W, Sarlomo-Rikala M, Andersson LC, Knuutila S, Miettinen M: DNA sequence copy number changes in gastrointestinal stromal tumors: tumor progression and prognostic significance. Cancer Res 2000, 60:3899-3903.

8. Corless CL, Fletcher JA, Heinrich MC: Biology of gastrointestinal stromal tumors. J Clin Oncol 2004, 22:3813-3825.

9. Lasota J, Wozniak A, Kopczynski J, Dansonka-Mieszkowska A, Wasag B, Mitsuhashi T, Sarlomo-Rikala M, Lee JR, Schneider-Stock R, Stachura J, Limon J, Miettinen M: Loss of heterozygosity on chromosome 22q in gastrointestinal stromal tumors (GISTs): a study on 50 cases. Lab Invest 2005, 85:237-247.

10. Tornillo L, Terracciano LM: An update on molecular genetics of gastrointestinal stromal tumours. J Clin Pathol 2006, 59:557-563.

11. Heinrich MC, Rubin BP, Longley BJ, Fletcher JA: Biology and genetic aspects of gastrointestinal stromal tumors: KIT activation and cytogenetic alterations. Hum Pathol 2002, 33:484-495.

12. Lungu $\mathrm{O}$, Wright $\mathrm{TC} \mathrm{Jr}$, Silverstein S: Typing of human papillomaviruses by polymerase chain reaction amplification with $\mathrm{L} 1$ consensus primers and RFLP analysis. Mol Cell Probes 1992, 6:145-152.

13. Mullenbach R, Lagoda PJ, Welter C: An efficient salt-chloroform extraction of DNA from blood and tissues. Trends Genet 1989, 5:391.

14. Penzel R, Aulmann S, Moock M, Schwarzbach M, Rieker RJ, Mechtersheimer G: The location of KIT and PDGFRA gene mutations in gastrointestinal stromal tumours is site and phenotype associated. $J$ Clin Pathol 2005, 58:634-639.

15. Daum O, Grossmann P, Vanecek T, Sima R, Mukensnabl P, Michal M: Diagnostic morphological features of PDGFRA-mutated gastrointestinal stromal tumors: molecular genetic and histologic analysis of 60 cases of gastric gastrointestinal stromal tumors. Ann Diagn Pathol 2007, 11:27-33.

16. Kallioniemi A, Kallioniemi OP, Sudar D, Rutovitz D, Gray JW, Waldman F, Pinkel D: Comparative genomic hybridization for molecular cytogenetic analysis of solid tumors. Science 1992, 258:818-821.

17. Ribeiro FR, Jeronimo C, Henrique R, Fonseca D, Oliveira J, Lothe RA, Teixeira MR: 8q gain is an independent predictor of poor survival in diagnostic needle biopsies from prostate cancer suspects. Clin Cancer Res 2006, 12:3961-3970.

18. Kirchhoff M, Gerdes T, Rose H, Maahr J, Ottesen AM, Lundsteen C: Detection of chromosomal gains and losses in comparative genomic hybridization analysis based on standard reference intervals. Cytometry 1998, 31:163-173.

19. ISCN (2005): An International System for Human Cytogenetic Nomenclature. Edited by: Tommerup N, Shaffer LG. Basel: S. Karger; 2005.

20. Demetri GD, Benjamin RS, Blanke CD, Blay JY, Casali P, Choi H, Corless CL, Debiec-Rychter M, Dematteo RP, Ettinger DS, Fisher GA, Fletcher CD, Gronchi A, Hohenberger P, Hughes M, Joensuu H, Judson I, Le Cesne A, Maki RG, Morse M, Pappo AS, Pisters PW, Raut CP, Reichardt P, Tyler DS, Abbeele AD Van den, von Mehren M, Wayne JD, Zalcberg J: NCCN Task Force report: management of patients with gastrointestinal stromal tumor (GIST) - update of the NCCN clinical practice guidelines. J Natl Compr Canc Netw 2007, 5:S1-29.

21. Veiga I, Silva M, Veira J, Pinto C, Pinheiro M, Torres L, Soares M, Santos L, Coutinho C, Dinis J, Costa N, Guimaraes dos Santos J, Lopes P, Lopes C, Teixeira M: Hereditary gastrointestinal stromal tumors sharing the KIT exon 17 germline mutation $p . A s p 820 T y r$ develop through different cytogenetic progression pathways. Genes Chromosomes Cancer 2010, 49:91-98.

22. Joensuu H: Risk stratification of patients diagnosed with gastrointestinal stromal tumor. Hum Pathol 2008, 39:1411-1419.

23. Fletcher CD, Berman JJ, Corless C, Gorstein F, Lasota J, Longley BJ, Miettinen M, O'Leary TJ, Remotti H, Rubin BP, Shmookler B, Sobin LH Weiss SW: Diagnosis of gastrointestinal stromal tumors: A consensus approach. Hum Pathol 2002, 33:459-465.

24. Yang J, Du X, Lazar AJ, Pollock R, Hunt K, Chen K, Hao X, Trent J, Zhang W: Genetic aberrations of gastrointestinal stromal tumors. Cancer 2008, 113:1532-1543.
25. Rubin BP: Gastrointestinal stromal tumours: an update. Histopathology 2006, 48:83-96.

26. Gomes AL, Gouveia A, Capelinha AF, de la CD, Silva P, Reis RM, Pimenta A, Lopes JM: Molecular alterations of KIT and PDGFRA in GISTs: evaluation of a Portuguese series. J Clin Pathol 2008, 61:203-208.

27. Martin J, Poveda A, Llombart-Bosch A, Ramos R, Lopez-Guerrero JA, Garcia del Muro J, Maurel J, Calabuig S, Gutierrez A, Gonzalez de Sande JL, Martinez J, De Juan A, Lainez N, Losa F, Alija V, Escudero P, Casado A, Garcia P, Blanco R, Buesa JM: Deletions affecting codons 557-558 of the C-KIT gene indicate a poor prognosis in patients with completely resected gastrointestinal stromal tumors: a study by the Spanish Group for Sarcoma Research (GEIS). J Clin Oncol 2005, 23:6190-6198.

28. Lasota J, Miettinen M: Clinical significance of oncogenic KIT and PDGFRA mutations in gastrointestinal stromal tumours. Histopathology 2008, 53:245-266.

29. Lux ML, Rubin BP, Biase TL, Chen CJ, Maclure T, Demetri G, Xiao S, Singer S, Fletcher CD, Fletcher JA: KIT extracellular and kinase domain mutations in gastrointestinal stromal tumors. Am J Pathol 2000, 156:791-795.

30. Wardelmann E, Losen I, Hans V, Neidt I, Speidel N, Bierhoff E, Heinicke T, Pietsch T, Buttner R, Merkelbach-Bruse S: Deletion of Trp-557 and Lys558 in the juxtamembrane domain of the c-kit protooncogene is associated with metastatic behavior of gastrointestinal stromal tumors. Int $J$ Cancer 2003, 106:887-895.

31. Cho S, Kitadai Y, Yoshida S, Tanaka S, Yoshihara M, Yoshida K, Chayama K: Deletion of the KIT gene is associated with liver metastasis and poor prognosis in patients with gastrointestinal stromal tumor in the stomach. Int J Oncol 2006, 28:1361-1367.

32. Debiec-Rychter M, Cools J, Dumez H, Sciot R, Stul M, Mentens N, Vranckx H, Wasag B, Prenen H, Roesel J, Hagemeijer A, Van Oosterom A, Marynen $P:$ Mechanisms of resistance to imatinib mesylate in gastrointestinal stromal tumors and activity of the PKC412 inhibitor against imatinibresistant mutants. Gastroenterology 2005, 128:270-279.

33. Wardelmann E, Hrychyk A, Merkelbach-Bruse S, Pauls K, Goldstein J, Hohenberger P, Losen I, Manegold C, Buttner R, Pietsch T: Association of platelet-derived growth factor receptor alpha mutations with gastric primary site and epithelioid or mixed cell morphology in gastrointestinal stromal tumors. J Mol Diagn 2004, 6:197-204.

34. Rubin BP, Heinrich MC, Corless CL: Gastrointestinal stromal tumour. Lancet 2007, 369:1731-1741

35. Sandberg AA, Bridge JA: Updates on the cytogenetics and molecular genetics of bone and soft tissue tumors. gastrointestinal stromal tumors. Cancer Genet Cytogenet 2002, 135:1-22.

36. Gunawan B, von Heydebreck A, Sander B, Schulten HJ, Haller F, Langer C, Armbrust T, Bollmann M, Gasparov S, Kovac D, Fuzesi L: An oncogenetic tree model in gastrointestinal stromal tumours (GISTs) identifies different pathways of cytogenetic evolution with prognostic implications. J Pathol 2007, 211:463-470.

37. Andersson J, Sjogren H, Meis-Kindblom JM, Stenman G, Aman P, Kindblom LG: The complexity of KIT gene mutations and chromosome rearrangements and their clinical correlation in gastrointestinal stromal (pacemaker cell) tumors. Am J Pathol 2002, 160:15-22.

38. Assamaki R, Sarlomo-Rikala M, Lopez-Guerrero JA, Lasota J, Andersson LC, Llombart-Bosch A, Miettinen M, Knuutila S: Array comparative genomic hybridization analysis of chromosomal imbalances and their target genes in gastrointestinal stromal tumors. Genes Chromosomes Cancer 2007, 46:564-576

39. Wozniak A, Sciot R, Guillou L, Pauwels P, Wasag B, Stul M, Vermeesch JR, Vandenberghe P, Limon J, Debiec-Rychter M: Array CGH analysis in primary gastrointestinal stromal tumors: cytogenetic profile correlates with anatomic site and tumor aggressiveness, irrespective of mutational status. Genes Chromosomes Cancer 2007, 46:261-276.

Pre-publication history

The pre-publication history for this paper can be accessed here: http://www.biomedcentral.com/1741-7015/8/26/prepub

doi: $10.1186 / 1741-7015-8-26$

Cite this article as: Silva et al., Chromosome copy number changes carry prognostic information independent of KIT/PDGFRA point mutations in gastrointestinal stromal tumors BMC Medicine 2010, 8:26 